Artikel

Julia Prager*

\title{
Vor-Schriften? Regieanweisungen als (Teil von) Notationen in Elfriede Jelineks Theatertexten
}


Abstract: Der Beitrag folgt dem Vorhaben, „Regieanweisungen" in Elfriede Jelineks Theatertexten als "szenische Stellen" zu verhandeln. Diese nehmen Vermittlungs- oder Übersetzungsfunktionen zwischen der als "Autorin" inszenierten persona und der Institution Theater einerseits sowie zwischen Text und Aufführung andererseits ein. Um die Verfahren und Funktionsweisen von Jelineks "szenischen Stellen" herauszuarbeiten, greift der Beitrag auf eine von der "Notation" aus gerichtete Forschungsperspektive zurück. Eine solche Fokussierung ermöglicht es, so lautet die grundlegende Annahme, Übersetzungsleistungen zwischen Schrift, Stimmen und Klängen sowie damit verkoppelte Problematiken der Begrenzung und Potenz von Aufschreibe- wie auch Aufführungspraktiken in ihren soziopolitischen, medialen und historischen Kontexten vordergründig werden zu lassen. Hierfür kommen zunächst Metaphoriken (Echokammer, Partitur, Poly- und Heterophonie) in den Blick, die vielfach zur Beschreibung von Jelineks Texten dienen. Jelineks Textverfahren werden als Übersetzungspraxis zwischen Notation und Klangphänomenen ausgewiesen, die Brüche in der medialen und kulturellen Übertragung mitverhandelt. Der Beitrag spricht sich in diesem Sinne dafür aus, das Heterophone in der Beschreibungssprache zu bevorzugen, um Jelineks Praxis des Übersetzens begrifflich einzuholen. Im anschließenden Abschnitt werden Modulationen von "szenischen Stellen" in Jelineks Stücken als "kleine Formen" untersucht, um eine Ausdifferenzierung der komplexen medialen Operationen ihrer "notierenden" Textverfahren anzuregen.

The article follows the attempt to negotiate "stage directions" in Elfriede Jelinek's theater texts as "scenic passages". These take on mediating or translating functions between the persona staged as "author" and the institution of theater on the one hand, and between text and performance on the other. To elaborate the modes of operation of Jelinek's "scenic passages," the article resorts to a research perspective directed from the field of "notation." The basic assumption is that such a focus makes it possible to foreground translation processes between writing, voices, and sounds, as well as the interconnected problematics of the limitation and potency of notation in their socio-political, medial, and historical contexts. For this purpose, metaphors (echo-chamber, score, poly- and heterophony), which often serve to describe Jelinek's texts, are first brought into view. Jelinek's textual procedures are shown to be a practice of translation between notation and sound phenomena, which negotiates ruptures in medial and cultural transmission. In this sense, the paper argues for privileging the heterophonic in descriptive language in order to conceptually catch up with Jelinek's practice of translation. In the subsequent section, modulations of "scenic passages" in Jelinek's plays are examined as "small forms" in order to stimulate a differentiation of the complex medial operations of her "notating" textual procedures.

Keywords: Elfriede Jelinek, Theatertexte, Notation, Heterophonie, kleine Formen, szenische Stellen

*Dr. Julia Prager, Technische Universität Dresden, Institut für Germanistik, Professur für Medienwissenschaft und Neuere deutsche Literatur, julia.prager@tu-dresden.de

Nicht Bei-, nicht Nebentext, keine Anweisung an das Theater (weder an Regie noch Spielende), sondern Teil des Textes, Stimme der figurgewordenen "Autorin"1 sollen sie sein, die mehr oder weniger tentativen, aufdringlich imperativen wie

1 Vgl. u. a. Jelinek, Elfriede (2002a): Ein Sportstück. Reinbek bei Hamburg: Rowohlt, S. 7. auch beiläufig kommentierenden, monströs ausufernden oder bis zum eigenen Verschwinden verkleinerten Text-Stellen, die in Elfriede Jelineks Theatertexten an die Stelle von Regieanweisungen treten. In einem Interview mit Theater heute von 1992 gibt Jelinek an, weniger für eine Theaterpraxis zu schreiben als für die "Idee eines Ortes, wo man Sprache und Figuren öffentlich aus- 
stellen kann"; nicht für ein Theater also, sondern für "das Theater".2 Noch im gleichen Atemzug prangert sie das patriarchal strukturierte Gefüge eines Theaterbetriebs an, der auch seine Autorinnen auf ihren zugewiesenen Platz verweist. ${ }^{3}$ Mit Blick auf die damalige wie auch gegenwärtige Dominanz an männlichen Regisseuren (die Jelinek nicht zuletzt selbst als Wunschkandidaten für die Inszenierung ihrer Stücke ins Spiel brachte) ${ }^{4}$ scheint eine derart kritische Beanstandung immer noch nicht passé. Wenn die Stimme der "Autorin" in manchen dieser hinsichtlich ihrer Stellung und Bezeichnung ins Wanken geratenen Regieanweisungen konfrontativ appellativ auftritt - ein anderes Mal wie beiläufig von der Seite kommentiert oder trotzig aus dem off spricht -, dann zum Teil auch im impliziten Schlagabtausch mit vorangegangenen inszenatorischen Entwürfen, die verlustigende Figurationen Jelineks auf die Bühne zerrten: Unvergessen bleiben die Inszenierung von Raststätte oder Sie machens alle, in der Frank Castorf eine übergroße, wirre Worte sprechende und nicht zu stoppende Sexpuppe mit Jelineks Gesicht auf die Bühne stellte, oder auch die "Vagina-Dialoge", eine Szene aus Ulrike Maria Stuart, in der Nicolas Stemann die "Autorin" als Plüsch-Vagina auftreten ließ.

Jelineks für das Theater geschriebene Texte führen ein über die Begrenzung der jeweiligen Stücke hinausreichendes Streitgespräch mit dem Theater als Institution - eben nicht zuletzt ausgetragen über die verschieden gestalteten szenischen Anweisungen. Aus einer solchen Perspektive und entgegen der Jelinek so oft zugeschriebenen Indifferenz gegenüber szenischen Umsetzungen lässt sich ihre viel zitierte Vorschrift "Machen Sie was Sie wollen"5 als Spur eines Auf-

2 Becker, Peter von (1992): Wir LEBEN auf einem Berg von Leichen und Schmerz. In: Theater heute, 1992/9, S. 1-9, hier S. 2. Auch in der "Nachbemerkung" von Macht nichts wird betont, dass "diese Texte" nicht zuletzt in Bezug auf die an den Anfang gestellt unmöglich einzulösende ,szenische Stelle', dass eine tote Schauspielerin auftrete, zwar für das Theater geschrieben seien, aber nicht für eine Theateraufführung. Jelinek, Elfriede (2002b): Macht nichts. Eine kleine Trilogie des Todes. Reinbek bei Hamburg: Rowohlt, S. 85.

3 Becker, Peter von: Wir leben auf einem Berg, S. 2.

4 Insb. Einar Schleef, George Tabori, Christoph Marthaler, Frank Castorf, Christoph Schlingensief und Nicolas Stemann.

5 Jelinek, Elfriede: Ein Sportstück, S. 7. begehrens lesen. Denn, so hat auch Stemann mehrfach (ironisch) beklagt, wie man denn wissen können solle, was man wolle, und hinzugefügt: "Man kann es gar nicht oft genug sagen: Sie nerven, die Texte! Sie sind anstrengend und penetrant. Sie kämpfen, meinetwegen, doch sie kämpfen zutiefst unfair." 6

Der konfrontativ aufbegehrende und gleichermaßen bittstellende Appell, ${ }^{7}$ der sich an Regie wie Spielende sowohl über Form als auch Inhalt der ,szenischen Stellen' je verschieden richtet, ruft die Akteur*innen als Mittler zwischen Text und Theater an, das gemeinsame Spannungsverhältnis von Anziehung und Abstoßung derart produktiv werden zu lassen, dass transformative Übersetzungsprozesse in Gang kommen, aus denen beide Seiten als etwas Anderes hervorgehen.

Wenn die "Autorin" sich mittels dieser TextStellen als solche entmachtet ("Die Autorin gibt nicht viele Anweisungen, das hat sie inzwischen gelernt. "8), auf- und abtritt ("Die Autorin tritt hinkend und desolat wieder auf."9) oder sich selbst aus dem Spiel nimmt, noch bevor die*der Regisseur*in als "Autor*in" dazu tritt ("Die Autorin ist weg, sie ist nicht der Weg. ${ }^{10}$ ), dann scheint sie sich nicht einfach rauszuhalten, sondern vielmehr in anderer Form, etwa als Kommentatorin oder auch als geschwätzige Bühnen- wie Kostümbildnerin wieder einzumischen. ${ }^{11}$

6 Stemann, Nicolas: Das ist mir sowas von egal! Wie kann man machen sollen, was man will? - Über die Paradoxie, Elfriede Jelineks Theatertexte zu inszenieren. In: Theater heute. Arbeitsbuch 2006, S. 62-68, hier S. 62.

7 Komisch ausgestellt wird dies u. a. in der das Stück Das Licht im Kasten einleitenden, szenischen Stelle': Also, ich weiß jetzt gar nichts mehr, am liebsten ließe ich nur Plüschtiere, also Schauspielerinnen und Schauspieler als Plüschtiere auftreten, aber nie werde ich das bekommen! Das wollte ich für mein letztes Stück haben, ich finde nicht, daß das zuviel verlangt ist. Ich will es, ich will es. Aber Sie wollen mit Sicherheit was andres, bloß werden Sie Sicherheit dadurch nicht gewinnen. Also, wer auch immer, na und?" Jelinek, Elfriede: Das Licht im Kasten. Elfriede Jelineks Website: https://elfriedejelinek.com/flicht.htm. (31.01.2021).

8 Jelinek, Elfriede: Sportstück, S. 7.

9 Ebd., S. 184.

10 Jelinek, Elfriede: Nachbemerkung. In: Jelinek, Elfriede: Macht nichts, S. 85-90, hier S. 90.

11 Vgl. hierzu auch Wirth, Uwe (2010): Lob der Oberfläche! Der Tod und die Mode in Elfriede Jelineks, Jackie'. In: Eder, Thomas/Vogel, Juliane (Hg.): Lob der Oberfläche. Zum Werk von Elfriede Jelinek. München: Wilhelm Fink, S. 71-85. 
In eben dieser Weise lässt sich auch nicht einfach behaupten, die in den frühen Stücken noch markierte Differenzierung von Regieanweisung und Sprechtext würde zusammen mit den Figuren im Laufe der Jahre sukzessive abgeschafft. Eher wird ihre andauernde Transformation beobachtbar, die sich im Besonderen auf der Szene der Schrift abspielt: Die anfänglich zumeist vom Sprechtext der auftretenden Figuren abgesetzten, vielfach kursiv gestalteten Anweisungen (z. B. Burgtheater, Der Tod und das Mädchen I-V) werden später, beispielsweise in Ulrike Maria Stuart, im fettgedruckten Schriftbild mit den Figurennamen verschmolzen. Aber auch wenn keine Auftritte mehr gekennzeichnet sind, weil abgrenzbare Figuren fehlen, sondern lediglich Sprechabsätze den Fließtext unterbrechen - wie etwa im jüngsten Stück Schwarzwasser - erscheinen szenische Anmerkungen in Form von Überschriften zugleich vergrößert und verkleinert. ${ }^{12}$ Und noch in jenen Texten, in denen nichts mehr auf die sich einmischende "Autorin" hinzudeuten scheint, drängt sich diese Absenz unvermeidlich als nachhallende Vorschrift auf: Machen Sie doch, was Sie wollen.

Das Aufbegehren, das sich sowohl in den ,szenischen Stellen' wie auch in den Texten und den sie hervorbringenden Zitationsverfahren immer wieder neu in Szene setzt und dabei gerade die Möglichkeit des Auf-die-Szene-Tretens, des Sichtund-hörbar-Werdens zur Verhandlung stellt, wird einmal mehr als Ringen um das Un-Vernehmbare beschreibbar. Denn grundsätzlich exponieren die thematischen Stränge und daran gekoppelten textuellen Verfahren von Jelineks Stücken die Prekarität von marginalisierten Stimmen, ihrer Vernehmbarkeit und des Gehör-Leihens. Dass es gerade Formen von lautstarkem, "ausgekotztem"13, nicht enden wollendem Aus-Sprechen heterogener Stimmen sind, die das Unvernehmen mehr ausstellen als einhegen, lässt sich als ein weiteres konfrontatives Verfahren beschreiben, das sich im Besonderen durch das Verhältnis von ,szenischen Stellen' und (anderem) Sprechtext ausdrückt und sich damit zwischen Text und Theater austrägt.

Um sich diesem Verfahren zu nähern, so möchte dieser Beitrag zeigen, erscheint eine vom

12 Jelinek, Elfriede (2020): Schwarzwasser. Am Königsweg. Zwei Theaterstücke. Reinbek bei Hamburg: Rowohlt. 13 Becker, Peter von: Berg von Leichen und Schmerz, S. 2. komplexen Feld der Notation aus gerichtete Perspektive vielversprechend. ${ }^{14}$ Denn sie ermöglicht es, Übersetzungsleistungen zwischen Schrift, Stimmen und Klängen sowie damit verkoppelte Problematiken der Begrenzung und Potenz von Aufschreibepraktiken in ihren jeweiligen soziopolitischen, medialen und historischen Kontexten vordergründig werden zu lassen.

Im folgenden Abschnitt sollen zunächst dem Bereich des Akustisch-Auditiven zuzurechnende Metaphoriken (Echokammer, Partitur, Poly- und Heterophonie) in den Blick kommen, die der bisherigen Forschung vielfach zur Beschreibung von Jelineks Texten dienen. Mit Bezug auf die an die Heterophonie gekoppelten Problematiken von Vernehmbarkeit und Verschriftlichung werden Jelineks Textverfahren verstärkt als heterophone lesbar gemacht, insofern diese als für ihr eigenes Verlustgeschehen aufmerksame Übersetzungspraxis von heterogenen Stimm- und Klangphänomenen begriffen werden können.

Im anschließenden Abschnitt werden exemplarische Modulationen von ,szenischen Stellen' in Jelineks Stücken als, kleine Formen' untersucht, um auf diese Weise eine Ausdifferenzierung der komplexen medialen Operationen ihrer ,notierenden' Textverfahren anzuregen. Szenen des Schreibens, der Schrift wie auch des (Vor-) Lesens und Sprechens von Texten, so lautet die grundlegende Annahme, geraten durch ,szenische Stellen' in eine konfliktuelle Beziehung, die Notation als durch und durch relationale Praxis menschlicher wie nicht-menschlicher Akteur*innen ausstellt.

Wenn Jelinek Roland Barthes in einer ,szenischen Stelle' zum „Mitarbeiter"15 erklärt, möchte sich der vorliegende Beitrag dieses Verhältnis zunutze machen, um in seinem Vorgehen Berührungs- und Abstoßungspunkte beider notierend Schreibender für den Zusammenhang von stimmlicher Notation und deren Aufführung produktiv werden zu lassen.

14 Vgl. hierzu auch die Arbeiten im Rahmen des interdisziplinären Forschungsprojektes "Notation und Aufführung" des Interuniversitären Forschungsverbundes Elfriede Jelinek: https://www.ifvjelinek.at/forschungsarbeiten/projekt-notation-und-auffuehrung/. (23.03.21).

15 Jelinek, Elfriede: Der Tod und Das Mädchen I-V. Berlin: BvT, S. 65. 


\section{Echokammer und Partituren: Versuchsanordnungen des Heterophonen}

Einleitend zum Abschnitt "Kompositionen, Texte für Kompositionen, Libretti" vermerkt Pia Janke im Handbuch Elfriede Jelinek zum Verhältnis von Musik und Text: "Ist das ,Musikalische' in Jelineks Werken möglicherweise in den Aktivierungsformen des klanglichen Potenzials der einzelnen Sprachpartikel aufzuspüren, so muss eine Beschreibung des ,Musikalischen' ihrer Arbeiten jedoch im Bereich des Metaphorischen verbleiben."16 Damit formuliert sie insbesondere eine durchaus berechtigte Skepsis an wissenschaftlichen Bezugnahmen, die das vermeintlich Musikalische von Jelineks Texten biografistisch begründen. Gleichzeitig legt Jelinek selbst in eine solche Richtung führende Fährten, wenn sie sich etwa in Interviews dezidiert als Musikerin vorstellt oder Komponist*innen einen privilegierten Leseeindruck ihrer Texte zuschreibt. ${ }^{17}$ An anderer Stelle zieht sie sich jedoch wieder von einem Kurzschluss zwischen Komposition und literarischem Schreiben zurück, wenn sie den „entsetzlichen Abstraktionsvorgang" der musikalischen Notation als Grund dafür nennt, nicht weiter komponiert zu haben. ${ }^{18}$ Oder aber sie distanziert sich gänzlich von der selbst instrumentalisierten Zuschreibung: „Ich kann ja nicht sagen, dass ich Musikerin je war." 19

Es bleibt also zu fragen, welche anderen Möglichkeiten eröffnet werden können, das Verhältnis von Stimmen bzw. Klängen und Textverfahren les- oder erfahrbar zu machen, die sich weder auf Biografie, noch auf eine literaturwissenschaftliche Reduktion des klanglichen Potenzials im Sinne

16 Janke, Pia (2013): Kompositionen, Texte für Kompositionen, Libretti. In: Dies. (Hg.): Jelinek Handbuch. Stuttgart/Weimar: J. B. Metzler, S. 228-236, hier S. 228.

$17 \mathrm{Vgl}$. Becker, Peter von: Wir leben auf einem Berg, S. 6 sowie Koelbl, Herlinde (1998): Im Schreiben zu Haus: wie Schriftsteller zu Werke gehen. Fotografien und Gespräche. München: Knesebeck, S. 64.

18 Heinrichs, Hans-Jürgen (2004): Gespräch mit Elfriede Jelinek. In: Sinn und Form 2004/6, S. 760-783, hier S. 774. 19 Štajner, Tamara (2012): Musiker sind Interpreten, Schriftsteller schaffen etwas. Elfriede Jelinek Forschungszentrum: http://www.elfriede-jelinek-forschungszentrum. com/wissenschaftsportale/musik/das-projekt/interviewmit-elfriede-jelinek/. (31.01.2021). seiner bloßen Metaphorik beschränken, sondern die Rupturen der medialen Übersetzung hervortreten lassen.

Gerade in Verbindung mit den spezifischen Intertextualitätskonzeptionen, ${ }^{20}$ die Jelineks Verfahren des Zusammenkommens und medialen Übersetzens von Stimmen umfassen sollen, ist es insbesondere die Barthes'sche Echokammer, die herangezogen wird, um einen sprachlichen Ausdruck für das in einen Text transformierte klangliche Phänomen des Nachhalls zu finden. Mit Blick auf die zahlreichen Bezugnahmen fällt auf, dass die - trotz der teilweise expliziten Hinwendung Jelineks zu Barthes - sehr divergierenden Verfahren beider Schreibender (und Musizierender) in der verkürzten Sichtweise stimmlicher Metaphorik zumeist beinahe nahtlos zusammengeschweißt werden. Barthes' Echokammer - die er selbst weniger theoretisch darlegt, denn in seiner spezifischen, zwischen Literatur und Theorie changierenden écriture entfaltet und nicht zuletzt mit einer eindringlichen Aufforderung des écoute verbindet - dient hierbei vielfach als in Abstraktion gebanntes Klang-Bild, um Jelineks Stimmverfahren mehr begrifflich als begreiflich zu machen. Dabei birgt gerade die Konfrontation beider Verfahren und der damit verknüpften Haltungen das Potenzial, klangliche Aktivierungsformen als solche in ihren Divergenzen und Bezugspunkten aufzuspüren.

In Barthes' experimenteller Autobiografie Über mich selbst wird als „Widerhallraum" eben jene textuelle Versuchsanordnung beschrieben, mittels der in den Ohren der Lesenden wie Schreibenden eine vielstimmige Klanglandschaft entsteht bzw. eine Echowirkung erzeugt wird. ${ }^{21}$ Hier wie auch an anderer Stelle (etwa in der letzten Figur von Die Lust am Text) bezieht Barthes diese Stimmen und Nach-Klänge auf eine von ihm entworfene und zugleich ersehnte, wie Ottmar Ette formuliert, "Ästhetik der Lust": "Akustik und Stimme führen jene Körperlichkeit und jene Ästhetik der Lust in die Theorie wieder ein, die in Julia Kristevas Überführung der Bachtin'schen Vielstimmigkeit in eine vom Körper getrennte

20 Vgl. hierzu: Vogel, Juliane (2013): Intertextualität. In: Janke, Pia: Jelinek Handbuch, S. 41-46.

21 Barthes, Roland (2010): Über mich selbst. Berlin: Matthes und Seitz, S. 81-87. 
Intertextualität im Grunde für die Avantgarde verloren schien." 22

Nun scheint aber diese durchweg erotisierend gerahmte Lust in Barthes' akustisch-auditiver Umschrift von Intertextualität in deutlichem Gegensatz zum konfrontativen, aus gewaltvollen Prozessen hervorgegangenem, Stimmgewebe von Jelineks Texten zu stehen. Dies mag zunächst an den dort verwobenen Stimmen und den sie rahmenden Diskursen liegen: Während Barthes vornehmlich literarische Stimmen, solche der Theoriebildung wie auch jene geliebter Personen zusammenkommen und nachhallen lässt, sind die Stimmen, die sich in Jelineks Texten versammeln bzw. dort zusammengetrieben, aufgefangen, verstärkt werden - oder auch in ausgestellt dominierenden Stimmlagen untergehen - jene der unbetrauerten Toten, Ermordeten und Versehrten nationaler, kriegerischer, rassistischer, sexualisierter Verbrechen; marginalisierte Stimmen der Geflüchteten, Fliehenden und Vertriebenen.

Mehr noch führen die Texte vor, dass sie nicht einmal mehr Echokammern dieser absenten, unverfügbaren Stimmen sind, sondern solche ihrer medialen Präsentation. Und auch in der Aufnahme wie verzerrten Wiedergabe theoretischer oder literarischer Stimmen erzeugen Jelineks Texte kaum so etwas wie eine "Ästhetik der Lust". Vielmehr transformieren sie das Gefühl intimer Lust im Vernehmen von Stimmen in eine überaus komplexe Affektionsdynamik, in der Scham und Überforderung gleichermaßen evoziert werden wie Trauer und Wut, aber auch Formen überbordender Komik.

Eine von metaphorischem Gebrauch des Stimmlichen Abstand nehmende Perspektive stellt also weniger die Frage nach dem Ursprung der zusammenkommenden Stimmen - darin berühren sich Barthes' und Jelineks Verfahren -, denn nach der Transformation ihrer Klänge im Echoraum des Textes, des Ohres, des Gedankenraums. Tatsächlich schenken diesem Aspekt bisher nur vereinzelte Analysen Aufmerksamkeit. Beispielhaft kann hier auf Evelyn Annuß' Les- bzw. Hörweise verwiesen werden, etwa die Stimmgemenge in Wolken.Heim. als Raunen vernehmbar werden zu lassen, welches sich mit dem kreatürlich schmerzlichen Schrei der ausgerisse-

22 Ette, Ottmar (2016): Roland Barthes zur Einführung. Hamburg: Junius, ebook: Position 1950. nen Alraune verbindet. ${ }^{23}$ Und auch Eva Kormann wendet sich dem konkreten Klangphänomen Stimme bzw. seiner Transformation zu, wenn sie in der medialen Echokammer von Bambiland ein exzessives Rauschen ausmacht, das einer verstörenden Verstärkung der Medienflut gleichkommt, die ununterbrochen den Krieg, die Folterungen von Abu Ghraib nur vermeintlich näherbringen, sondern eher noch hinter die Oberfläche des Screens verbannen, um davon abzuschirmen. ${ }^{24}$ Stimmlicher Klang wird hier zu noise oder zum unvernehmbaren, weil im medialen (auch mythischen) Rahmen gefangenen, Auf-Schrei.

Annuß spricht sich in diesem Zusammenhang dafür aus, das Heterophone in der Beschreibung von Jelineks Textverfahren zu bevorzugen.25 Während literaturwissenschaftliche Bezugnahmen auf Mehr- und Vielstimmigkeit zumeist textbasierte Konzeptionen (etwa jene von Bachtin) aufgreifen, verweist das musikethnologische Feld der Heterophonie konkret auf Problematiken des Gegenstimmlichen, des Unvernehmbaren sowie auf solche der Notation, die Jelineks Schreibverfahren auszutragen scheinen: Wörtlich Anders- oder Fremdstimmigkeit meinend, geht der Ausdruck "Heterophonie" vermutlich auf Platon zurück, der in seinen Gesetzen (VII 812d) wohl diesen Neologismus prägte, um eine bestimmte von Berufsspielern angewandte Technik (die er im Übrigen zwar missbilligte, aber einem höheren künstlerischem ,Stadium' zuordnete als viele nachkommende Musikethnolog*innen $)^{26}$ zu beschreiben, bei der ein Instrument mitsamt einer eigenen Melodie zur Singstimme hinzutritt.

In der historischen Musikethnologie wurde die Heterophonie insbesondere mit nicht-westli-

23 Annuß, Evelyn (2005): Theater des Nachlebens. München: Wilhelm Fink, S. 145.

24 Kormann, Eva (2009): Die Bühne als medialer EchoRaum. Zu Elfriede Jelineks Bambiland. In: Schößler, Franziska (Hg.): Ökonomie im Theater der Gegenwart. Ästhetik, Produktion, Institution. Bielefeld: transcript.

25 Annuß, Evelyn: Theater des Nachlebens, S. 139.

26 Neubecker, Annemarie Jeanette (1992): Altgriechische Musik 1958-1986. In: Gärtner, Hans/Petersmann, Hubert (Hg.): Lustrum. Internationale Forschungsberichte aus dem Bereich des klassischen Altertums. Göttingen: Vandenhoeck \& Ruprecht, S. 99-176, hier S. 142 sowie Weinbuch, Isabell (2010): Das musikalische Denken und Schaffen Carl Orffs. Ethnologische und Interkulturelle Perspektiven. Mainz u. a.: Schott Campus, S. 138. 
cher Musik, etwa mit den Gamelanorchestern in Verbindung gebracht und zum Teil im Sinne einer "nicht rationalen Mehrstimmigkeit" 27 aufgefasst. In der überarbeiteten achten Auflage von Karl Wörners Geschichte der Musik von 1993 wird der Versuch, die schriftlose heterophone Musik der Gamelanorchester notenschriftlich darzustellen, als "irreführendes Bild einer Partitur wie in der abendländischen Mehrstimmigkeit, zu der sie jedoch keinerlei Bezüge" aufweise, beschrieben. ${ }^{28}$

Mit diesen Verweisen auf das an die Heterophonie gekoppelte "Andersartige", "Irrationale" und sich einer Verschriftlichung Entziehende lassen sich Jelineks Textverfahren eben insofern als heterophone fassen, als sie sich einer dokumentarischen oder darstellenden Vorstellung von Notation widersetzen. Vielmehr werden sie als eine performative Versuchsanordnung von nicht zur Ruhe kommenden Übersetzungsprozessen begreifbar, die das notwendige Scheitern an dem nicht einzuholenden Unvernehmen der anderen Stimmen selbst exponieren.

Aus einer solchen Perspektive nehmen die ,szenischen Stellen' eben auch jene Position behelfsmäßiger Zusatzzeichen, Vermerke oder (Rand-)Notizen ein, die der brüchigen Notation eingetragen sind, um in der Überantwortung des Textes an zukünftige Aufführungen das Verlustgeschehen selbst einzufordern - wie auch immer diese letztlich gestaltet sein mögen. Eine solche Beanstandung des Unvernehmens drängt sich u. a. in der ersten, das Stück eröffnenden, ,Anweisung' von Abraumhalde auf:

(Also, ihr macht eh, was ihr wollt. Trotzdem sage ich, wie ich es mir vorstelle. Wie immer habe ich die Ästhetik der Aktionen von Paul McCarthy im Auge und nicht nur dort. „Bunker Basement" oder "Piccadilly Circus" wären Beispiele dafür, was ich meine. Der Text könnte vielleicht nichts als Hintergrundmusik, eine Tapete für irgendwelche Popanze sein. Er läuft sozusagen durch als eine Endlosschleife (wenns aus ist, fängt es wieder von vorne an) - ich könnte mir sogar vorstellen, daß er, beinahe unhörbar, wie eine Litanei als Hintergrund zu einer Aufführung von "Nathan" (oder einem ähnlichen Stück) durchläuft, vielleicht sogar von mir selber

27 Wörner, Karl H. (1980): Geschichte der Musik. Ein Studien- und Nachschlagewerk. 7. Aufl. Göttingen: Vandenhoeck \& Ruprecht, S. 8.

28 Wörner, Karl H. (1993): Geschichte der Musik. Ein Studien- und Nachschlagewerk. 8. Aufl., bearb. v. Wolfgang Gratzer u. a. Göttingen: Vandenhoeck \& Ruprecht, S. 8. gelesen, manchmal etwas lauter, an der Hörgrenze, vielleicht sogar darüber, das müßte jemand vom Pult aus steuern, dann wieder unhörbar, man kann sich von ihm auch beliebige Stücke abschneiden, ungefähr wie von einer langen Wurst. Man kann Sätze auch mittendrinnen durchschneiden. Falls man es inszenieren möchte: Die Figuren, die sprechen, sollen entweder vergrößert werden, vielleicht durch riesige Pappmachéköpfe, die sie tragen, am besten mit dem Gesicht nach hinten, sodaß sie auf der Bühne dauernd zusammenstoßen, umfallen, das Bühnenbild, falls es eins gibt, umreißen, die Bühne auf unterschiedlichste Weise devastieren, etc. etc. Oder die Figuren verdoppeln sich auf andere Weise. Sie tragen Kopf und Glieder, sozusagen ein zweites Mal, eben verdoppelt, mit sich herum. Der Kopf soll dann ihre Gesichtszüge tragen, er kann aber auch andre haben. Es soll eine Vermehrung und/oder allgemeine Vergrößerung von allem stattfinden. Vielleicht, wenn sich Gegenstände auf der Bühne befinden, sollen die proportional sehr klein sein, damit die Figuren riesig wirken. Der Text kann aber auch, wie gesagt, als reine Geräuschtapete eingesetzt werden, sehr leise und beinahe unverständlich, wie fernes Gemurmel, und nur manchmal wirklich hörbar und verständlich. $)^{29}$

Bei dieser ausufernden, hinsichtlich ihrer Platzierung in der räumlichen An-Ordnung des Textes als Vor-Schrift fungierenden Textstelle fällt zunächst die trotzige und so gar nicht indifferent auftretende Bemerkung "[I]hr macht eh was ihr wollt. Trotzdem sage ich, wie ich es mir vorstelle" auf. Was folgt, ist eine Kaskade von fragmentarischen Vorstellungsbildern und Klangräumen, die sich gegenseitig Konkurrenz machen oder in ihrem Nebeneinander Zufälligkeit ausstellen. Dabei treten jedoch nicht allein die hier notierten Vorstellungen zueinander in Konkurrenz, sondern der Text fordert auch die Konfrontation mit seinem Medienwechsel als Verlustgeschehen heraus, indem er dazu auffordert, als gemurmelte "Litanei", als „Geräuschtapete" und „Hintergrundmusik" manchmal knapp über der „Hörgrenze”, manchmal "unhörbar" von einer elektronisch eingespielten Stimme der lesenden „Autorin", sogar zerschnitten, aber dafür in Form einer Endlosschleife regelrecht abgespielt zu werden. Stemann trug diesem szenischen Appell an das Unvernehmbare des Textes und seiner Stimmen im Übrigen Rechnung, als er eine halbe Stunde lang den in Abraumhalde transformiert nach-

29 Jelinek, Elfriede (2009): Abraumhalde. Elfriede Jelineks Website: https://www.elfriedejelinek.com/farhalde. htm. (31.01.2021). 
hallenden Nathan aus Lessings Fassung mittels eines aus dem Bühnenhimmel abgeseilten Lautsprechers hörbar werden ließ, während der Bühnenraum ansonsten leer blieb.

Besonders eindrucksvoll gestalten auch jene ,szenischen Stellen' das Ringen um die Anerkennbarkeit des Unvernehmbaren, die in ihrer Verkoppelung mit dem, anderen' Text das V. Prinzessinnendrama (Die Wand) enden lassen: Zunächst erfolgt eine ,szenische Stelle', die durch das eingesetzte Präsens auffällig konstativ markiert wird und dabei eher wie die Beschreibung einer performativen Vorstellung wirkt als eine an zukünftige Inszenierungen gerichtete Vor-Schrift: "(Aus einem altmodischen Kofferradio hören wir, von einer eifrigen Männerstimme gelesen)"30. Es folgt eine 23-zeilige Textstelle aus Homers Odyssee, die sechs Mal durch den die Lesenden wie Schauenden adressierenden Einschub "Das haben Sie ja gesehen, meine Damen und Herren" unterbrochen wird. Das angeblich Gesehene, auf das die Sprechstimme einschwört, wird hier jedoch lediglich als Erzählung präsentiert, die zudem noch durch ein "altmodisches" (rauschendes?) Medium vermittelt wird. Damit aber nicht genug. Es folgt eine weitere ,szenische Stelle', die das bereits in Frage stehende gehörte Gesehene nun vollkommen an die Grenze der Vernehmbarkeit verweist: "(Gleichzeitig, sehr leise, hören wir, unbedingt auf Altgriechisch!, mit zarter Frauenstimme, vielleicht ein Kind, eine Schülerin aus einer Stadt, toll wäre es, wenn die Schauspielerinnen, oder wenigstens eine von ihnen, aus der Theogonie des Hesiod (155ff) die folgenden netten Worte sprechen oder lesen könnte:)"31. Nicht nur wird ein anderer Text gleichzeitig gesprochen, er soll auch noch - entgegen der auf Deutsch gehaltenen Fassung im Theatertext - auf Altgriechisch vorgetragen werden. Auch wenn die weibliche, sogar piepsige Stimme womöglich die männliche, aus der Vergangenheit nachhallende Stimme nicht übertönt, so muss sie diese doch merklich stören. Der Anweisung folgend umspielt sie nicht, sondern entwickelt eine eigene, für den Großteil des Publikums zwar semantisch unvernehmbare, aber sich zum Hören an- und darbietende Stimme. Derart heterophon konterkariert sie die persuasive Stimme, die das Sehen und damit

30 Jelinek, Elfriede: Der Tod und Das Mädchen I-V, S. 140. 31 Ebd., S. 141-142. verbunden auch Evidenz zu erzeugen sucht. Sie appelliert an eine andere Form der Zeugenschaft, an das Gehör-Leihen als ein Offen-Bleiben für das andere, ${ }^{32}$ das sich dem Wissbaren entzieht - wie eben auch die Stimme der ,Regieanweisung' von einer scheinbaren Beschreibungssprache plötzlich in einen (durch das parenthetisch eingeschobene "toll wäre es" angezeigten) appellativen Modus wechselt.

\section{2 ,Kleine Formen' der Notation: Notizen, Kommentare, Merk- und Denkzettel}

Auch wenn sich Jelineks Theater-Texte nicht umstandslos als „Partituren für eine Inszenierung auf der Bühne" 33 fassen lassen, bietet sich doch das Nachdenken über Implikationen und Rupturen von Partituren an, um die medial spezifischen Operationen von ,szenischen Stellen' in Jelineks Theaterstücken auszudifferenzieren. Werden diese Textteile als in die ,Partitur' des Stückes eingetragene Notizen, Anmerkungen oder Kommentare aufgenommen - wie dieser Abschnitt versuchsweise vorführt - dann steht weiterhin infrage, inwiefern sich diese, kleine[n] Formen'34 von Notationen zu den anderen Textteilen verhalten. Denn aufgrund ihrer ex-zitierenden Verfahren, ${ }^{35}$ die sich eben als solche eines auditivakustischen Nachhallens beschreiben lassen, entziehen sich Jelineks Texte immer schon der ,großen Form'. Sie sprechen vielmehr ein Misstrauen an Meta- und Großnarrativen aus. Insbesondere die für das Theater geschriebenen Texte bleiben eben ,Stücke', Teile eines nicht zur Gänze kommenden Ganzen. ${ }^{36}$

32 Vgl.: Nancy, Jean-Luc (2004): Zum Gehör. Zürich/Berlin: diaphanes.

33 Kormann, Eva: Echo-Raum, S. 345.

34 De Mazza, Ethel Matala/Vogl, Joseph (2017): Graduiertenkolleg "Literatur- und Wissensgeschichte Kleiner Formen". In: Zeitschrift für Germanistik, 27/3, S. 579-585. JSTOR: www.jstor.org/stable/26583261. (31.01.2021).

35 Menke, Bettine (2001): Zitierfähigkeit: Zitieren als Exzitation. In: Gutenberg, Andrea/Poole, Ralph J. (Hg.): Zitier-Fähigkeit. Findungen und Erfindungen des Anderen. Berlin: Erich Schmidt, S. 153-171.

36 Vgl.: Nancy, Jean-Luc (1994): Die Kunst - Ein Fragment, übers. v. Jean-Pierre Dubost. In: ders. (Hg.): Bild- 
Demnach wird die Relation von Stück und Partitur dann produktiv, wenn diese nicht im chronologischen Sinn einer der Aufführung zeitlich vorgelagerten Vorschrift betrachtet, sondern auf ihre eigentümliche Zeitlichkeit hin befragt wird: Als Transaktionsdokumente, die in ihren Experimenten mit alphabetischen oder grafischen Zeichen nach einer Interpretation (Lesweise) oder Improvisation durch einen Ausführenden verlangen, nehmen Partituren Barthes zufolge weniger eine zukünftige Aufführung vorweg, als dass sie sich kontingenten Faktoren wie Zufall und Kontext überantworten. Der "Rolle des ,Interpreten'", so Barthes mit Blick auf die zeitgenössische postserielle Musik, werde abverlangt, dass dieser nun gewissermaßen zum "Mitautor der Partitur" werde, sie also mehr weiterschreibe als ausdrücke. ${ }^{37}$ Barthes veranschlagt damit die Zeitlichkeit von Partituren jenseits einer Dialektik von Ursprung und Wirkung. Alle zukünftigen Momente, die sich aus der Partitur heraus materialisieren, blicken durch die Abfolge aller früheren Aufführungen hindurch auf ihren ,Ursprung' zurück. Gleichzeitig wird die Partitur bereits auf ihre zukünftigen Verlebendigungen hin geschrieben wie auch - so ließe sich ergänzen - von vergangenen Notationen, Tonfolgen und Stimmlagen, Melodien etc. her geschrieben. ${ }^{38}$

Dieses Zusammenkommen von Vergangenheit und Zukunft in einer nicht "gegenwärtigen Gegenwart" ${ }^{19}$, das auch Jelineks Texte als komplexe Versuchsanordnungen von Notationen des Heterophonen konsequent ausstellen, betrifft allerdings nicht nur das Stück (und in seiner Aufführung das Verhältnis von Text und Theater), sondern auch die jeweilige räumliche Anordnung von Theatern: Als bauliche Vorrichtungen von Seh- und Hörräumen zeugen sie von vergangenen wie gegenwärtigen dispositiven Anordnungen, geografischen Lagen und ihren spezifischen gesellschaftlichen wie ästhetischen Zurichtun-

störung. Gedanken zu einer Ethik der Wahrnehmung. Leipzig: Reclam.

37 Vgl.: Barthes, Roland (2005): Vom Werk zum Text, übers. v. Dieter Hornig. In: ders.: Das Rauschen der Sprache. Frankfurt am Main: Suhrkamp 2005, S. 64-72, hier S. 71 .

38 Folkerts, Hendrik (2014): documenta 14: https://www. documenta14.de/de/south/464_der_partitur_nach_notation_verkoerperung_und_liveness (23.03.2021).

39 Kormann, Eva: Echo-Raum, S. 353. gen. Sie sind Echokammern aller aufgetretenen Stimmen, Sprachen und Stimmlagen und bleiben so ex negativo an die abwesenden und marginalisierten Stimmen, Sprachen, Akzente, Sozio- und Dialekte gekoppelt.

Anders als jene, in der schönen Schrift der Tante geschriebenen Anmerkungen oder nicht "ganz akkuraten Notizen" der Großmutter, die Barthes auf den Partituren in einem "großen Fach" im "kleinen Salon" entdeckt und ihm nostalgische Gefühle bescheren, ${ }^{40}$ fordern Jelineks als ,szenische Stellen' markierte Notizen, Anweisungen und Anmerkungen das generationenübergreifende Zusammenwirken von Stimmen auch in den jeweiligen Echokammern der Theater konfrontativ heraus.

Eindrücklich lässt sich dies am Beispiel Burgtheater zeigen, das nicht zuletzt Jelineks Wunsch nach am Wiener Burgtheater hätte uraufgeführt werden sollen. Eine derartige Konfrontation dieser beiden medial differenzierten, aber aufeinander bezogenen Echokammern steht jedoch bis heute aus. ${ }^{41}$

Hier bringt sich die an den Anfang des Stücks gesetzte ,szenische Stelle' regelrecht als Vorschrift in Stellung, und zwar sowohl im Sinne einer (um den Vorschlag einer Variation erweiterten) Anweisung als auch im Sinne einer der Aufführung zeitlich vorgelagerten Partitur:

Sehr wichtig ist die Behandlung der Sprache, sie ist als eine Art Kunstsprache zu verstehen. Nur Anklänge an den echten Wiener Dialekt! Alles wird genauso gesprochen, wie es geschrieben ist. Es ist sogar wünschenswert, wenn ein deutscher Schauspieler den Text wie einen fremdsprachigen Text lernt und spricht. ${ }^{42}$

Neben dem zur Kunstsprache transformierten Wiener Dialekt lässt das Stück weitere Stimmlagen, insbesondere ideologisch instrumentalisierte Sprechweisen des lieblich verklärenden „Burgtheaterdeutsch", anklingen. Die vorangestellte stimmliche Vorschrift verweist im ausgestellten

40 Samoyault, Tiphaine (2015): Roland Barthes: Die Biographie. Frankfurt am Main: Suhrkamp, S. 100.

41 Vgl. hierzu: Janke, Pia (2002): Die Nestbeschmutzerin. Jelinek und Österreich. Salzburg: Jung und Jung, S. 171 sowie Palm, Kurt (1986): Elfriede Jelinek. In: ders. (Hg.): Burgtheater. Zwölfelläuten. Blut. Besuchszeit. Vier österreichische Stücke. Berlin: Hentschel, S. 227-233, hier S. 229.

42 Jelinek, Elfriede (1982): Burgtheater. Elfriede Jelineks Website: https://www.elfriedejelinek.com/fburgth1.htm. (31.01.2021). 
Fremdwerden des lokalen Dialekts zunächst auf die prinzipielle Künstlichkeit dialektaler Sprachfärbungen inner- wie außerhalb des Theaters. Denn gerade nach Kriegsende wurde im Wiener Alltag eine regelrechte Kultivierung des Dialektalen gepflegt, um nicht zuletzt eine hörbare Abgrenzung gegenüber Deutschland und damit verbunden einer Beteiligung Österreichs an den nationalsozialistischen Verbrechen zu befördern. ${ }^{43}$ Sie exponiert aber auch jenen Kunstgriff, der jeder Notation von Oralität prinzipiell eingeschrieben ist.

Die exophone Strategie, das Fremdwerden der ,eigenen' Sprache zu provozieren, wird noch verstärkt, wenn die an Paula Wessely gekoppelte Figur Käthe im ersten Teil des Stücks von lieblich wienerisch akzentuiertem Dialekt, über seine ins Derbste getriebene Variation, in den "Burgtheaterton" verfällt, um dann immer mehr ins Stocken und Stammeln zu geraten: „Käthe ekstatisch, Burgtheaterton:

Dung! Dung! Dung! Karls Dung. Franzens Dung. Adolfs Dung. Gedüngte Hhherde. LIlleicheln unterm Baam. Marod... Krowot... Ttttorsch... Schschschood daß Ttttttortn! Tuatn. Henriette. Lavendel. Buxbaam. Morrrrdenrot! Junger Tutter! Hinreise. Au! Au! Auschschwww. . Schwester! Geh Pepperl, plausch net! ${ }^{44}$

Das Schriftbild nimmt das semantische Stottern in der Variation der Interpunktion auf und transformiert damit auch die phonetische Dimension der Schriftzeichen bzw. bleibt unklar, inwieweit sich phonetische Umsetzung der als Auslassungszeichen markierten Punkte von jenen drei durch zwei Spatien getrennten Punkten unterscheidet. Verbunden mit der szenischen Anweisung, dass alles "genauso gesprochen [wird], wie es geschrieben ist", führt das Textverfahren damit jenen von Gilles Deleuze beschriebenen Kunstgriff aus und vor, wonach die Sprache "so gespannt ist, dass sie zu stottern, murmeln, stammeln ... beginnt, sodass das Sprachliche insgesamt an eine Grenze [rührt], die dessen Außen hervortreten lässt und sich dem Schweigen aus-

43 Vgl.: Deutsch-Schreiner, Evelyn (2013): Burgtheater; Erlkönigin; Präsident Abendwind; Ich liebe Österreich; Das Lebewohl". In: Janke, Pia: Jelinek Handbuch, S. 137-146, hier S. 140.

44 Jelinek, Elfriede: Burgtheater. setzt." ${ }^{45}$ Das evozierte Schweigen, aber auch das Verstummen als differente Modi des Ausbleibens von Sprechen, die ihren Weg hier gerade über das unaufhörliche Aussprechen der Figuren nehmen, stellen einmal mehr eine Verbindung zu Fragen der Vernehmbarkeit des Unausgesprochenen und Unaussprechlichen her. Durch die Anweisung, am Text zu bleiben, um notwendig über inn zu stolpern, wird jedes habitualisierte, national und lokal gerahmte Sprechen (der deutschen Sprache und ihrer Variationen) deterritorialisiert und zugleich auf eine gemeinsame Geschichte verwiesen.

Mit Blick auf das Verhältnis von Text und Aufführung und die dabei ruptierend vermittelnden ,szenischen Stellen' lassen sich noch weitere Verfahren gleichzeitiger Zuwendung und Abkehr vom Text als Ursprung in Jelineks Theatertexten ausmachen. Im vorangegangenen Abschnitt wurden bereits ,Regieanweisungen' aus den Stücken Abraumhalde und (Die Wand) zitiert, in denen vorgeschlagen wird, bestimmte Stellen (sogar von der "Autorin" selbst) vorlesen zu lassen. Das Vorlesen scheint das zitierende Sprechen auf dem Theater sichtbar - möglicherweise auch hörbar - an den Text zurückzubinden. Dass das Textverfahren aber auch hier nicht eine Rehabilitation des Originals oder der Position der Autorin verfolgt, sondern derartige Konzeptionen weiter verschiebt, wird mit einem Seitenblick auf ein Interview noch verdeutlicht, in dem Jelinek das Vorlesen, besonders ein solches, das in Autor*innenlesungen performiert wird, regelrecht diskreditiert: "Niemand sollte vorlesen. Das ist ein Voyeurismus. Mir hat Ch. Enzensberger einmal gesagt, 68 haben wir einmal geglaubt, dass wir wenigstens die Dichterlesungen hätten abschaffen können." 46

In ihrer als Hörspiel bezeichneten Lesung Das Kommen wird diese Haltung nicht zurückgenommen - im Gegenteil: Jelinek liest hier in einer Weise, die in Intonation und Rhythmus an ein Schulkind erinnert und damit das Vorlesen als eben solches ausstellt. Auf diese Weise wird nicht

45 Deleuze, Gilles (2000): Stotterte er ..., übers. v. Joseph Vogl. In: ders.: Kritik und Klinik. Frankfurt am Main, S. 145-154, hier S. 152-153.

46 Maresch, Rudolf (1993): Nichts ist verwirklicht. Alles muss jetzt neu definiert werden. In: ders. (Hg.): Zukunft oder Ende: Standpunkte, Analysen, Entwürfe. Berlin: Boer, S. $125-143$. 
die Stimme der Autorin des Textes verlautbart, sondern vielmehr der, eigene' Text fremd. ${ }^{47}$

Nicht allein in Bezug auf die durch sie evozierten Relationen von Text und Theatern als Institutionen stellen die als, kleine Formen' beschreibbar werdenden ,szenischen Stellen' in Jelineks Theatertexten Verbindungen zwischen Medien, Körpern, Räumen und Zeiten her, die sich von einer bloßen „Intertextualität" abheben. Grundsätzlich sind ,kleine Formen' mit bestimmten Sammelaktivitäten verbunden bzw. operieren sie versammelnd. Sie treten nicht im Singular, sondern im Plural auf, wie sich überdeutlich an den variantenreichen Aufnahmen des "Machen Sie doch was Sie wollen"48 bzw. des "Also, ihr macht eh, was ihr wollt"49 ablesen lässt. Aber auch dann, wenn die ,szenischen Stellen' weniger als an die Regie gerichtete Anweisungen oder Anregungen erscheinen, sondern in Gestalt von plural adressierten Notizen, eröffnen die teilweise wie zufällige Gedanken notierten Stellen interwie außertextuelle Verweisräume. Beispielsweise stellt das notierte Vorstellungsbild der an Paul McCarthys Piccadilly Circus erinnernden Kostüme eine Relation zwischen den Stücken Ulrike Maria Stuart und Abraumhalde her, wobei das plurale Aufkommen dieser Imagination bzw. ihrer schriftgewordenen Notiz selbst noch kommentiert wird: "Wie immer habe ich die Ästhetik der Aktionen von Paul McCarthy im Auge und nicht nur dort."50 Dem online wiedergegebenen Ausschnitt von Ulrike Maria Stuart wurde noch ein photografischer Verweis auf Piccadilly Circus eingefügt,, der auf die Absenz der in diesem Textteil fehlenden schriftlichen Notiz des Vorstellungsbildes aufmerksam werden lässt.

Inwieweit sich solche Formen szenischer Notizen nicht an die tatsächliche szenische Umsetzung richten, sondern vor allem eine imaginative Vorstellungswelt mitschreiben, kann an unmöglich zu realisierenden Anregungen abgelesen werden, die etwa im I. Prinzessinnendrama (Schneewitt-

47 Jelinek, Elfriede: Das Kommen. ARD-Audiothek: https:// www.ardaudiothek.de/hoerspiel-pool/01-lesung-das-kommen-von-elfriede-jelinek/78745986. (31.01.20121).

48 Jelinek, Elfriede: Ein Sportstück, S. 7.

49 Jelinek, Elfriede: Abraumhalde.

50 Jelinek, Elfriede: Abraumhalde.

51 Jelinek, Elfriede (2005): Ulrike Maria Stuart. Elfriede Jelineks Website: https://www.elfriedejelinek.com/fstuart. htm. (31.01.2021). chen) dazu auffordern, dass keine menschlichen Schauspieler*innen den Text sprechen sollten, sondern zwei "riesige, popanzartige Figuren, die zur Gänze aus Wolle gestrickt und dann ausgestopft sind"52.

Mit Blick auf die Notiz als ,kleine Form' macht Barthes im Notizen-Machen jene Ausgangsbewegung des Schreibens aus, die das Form-Werden im Sinne eines fließenden Übergangs von der "Phantasie des Schreibens" zum "Begehren zu schreiben" hin zur "Praxis des Schreibens" ankündigt. ${ }^{53}$ Die Notiz dient hier nicht als Gedächtnishilfe, sich etwas, was einem entfallen könnte, zu notieren, sondern stellt den Versuch dar, eine Simultaneität des Niederschreibens mit dem Niedergeschriebenen herzustellen. Im Schreibfluss können sich Schreibende geradezu in den Fluss der Ereignisse begeben. Gegenwart, Augenblick würden demzufolge nicht notiert, sondern ge- bzw. erschrieben: "Man kann die Gegenwart schreiben, indem man sie aufzeichnet." 54 Insofern das Notieren hier als Schreibverfahren verstanden wird, ,einen Span des Gegenwärtigen abzuheben, aufzulesen, zu ergreifen, zu erbeuten, so wie er Ihnen vor Augen, ins Bewußtsein springt" 55 , setzt Barthes die Notiz (zeitweise) analog zum photografischen Schnappschuss. ${ }^{56}$ In der ,kleine Form' zeige sich der "Augenblick der Wahrheit", eine „Epiphanie". 57

Einmal mehr berühren sich hier Jelineks und Barthes' Vorstellungswelten, um dann völlig andere Bahnen einzuschlagen. Denn Jelineks in ,szenischen Stellen' mitnotierten visuelle wie akustische Vorstellungen, Imaginationen und Erinnerungen lassen sich weder an einen Entstehensprozess oder eine Idee zurückbinden, noch zeichnen sie die szenische Umsetzung vor. In jenen szenischen Notizen, die Sprechweisen, Kostüme, Ausstattung, Bühnenbild betreffen, drücken sich nicht Augenblicke der Wahrheit - das schnappschussartige ,Klick' der Schreibenden aus, sondern vielmehr eine medial evozierte Schreibpraxis. In eben dieser Weise illustriert das dem Online-Text von Ulrike Maria Stuart eingefügte Foto von McCarthys Performance nicht den

52 Jelinek, Elfriede: Der Tod und Das Mädchen I-V, S. 9. 53 Barthes, Roland (2008): Die Vorbereitung des Romans, Frankfurt am Main: Suhrkamp, S. 39-41; S. 211; S. 379-388. 54 Barthes, Roland: Vorbereitung des Romans, S. 54.

55 Barthes, Roland: Vorbereitung des Romans, S. 152.

56 Ebd., S. 154.

57 Ebd., S. 167-175. 
notierten Gedanken; es ist vielmehr der Gedanke im Sinne eines an ein Anderswo gekoppelten Vorstellungsbildes.

In Jelineks Textverfahren scheinen sich plurimediale Vorstellungen und instrumentelles Schreiben derart zu verschränken, dass sich überhaupt keine Abfolge des Schreibprozesses mehr ausmachen lässt. Darauf verweisen auch die immer wieder neu gewendeten paradoxalen Narrationen von Schreibszenen, die sie in verschiedenen Interviews entwirft: "Ich schreibe wahnsinnig schnell, weil ich Musikerin bin und schreibe, so schnell ich denken kann. Ich denke allerdings nicht sehr schnell. Inzwischen schreibe ich gleich in den Computer, weil das der Übermittlung zwischen den Gedanken und der Notation den geringst möglichen Widerstand entgegensetzt." 58 Und an anderer Stelle: "Ich habe immer gleich alles in den Rechner geschrieben. Von Hand mache ich bis heute höchstens Notizen, hauptsächlich auf alte Briefumschläge oder so. Ich muß mir immer alles gleich notieren, weil ich so ein schlechtes Gedächtnis habe. Das Tippen ist eine sehr organische Tätigkeit für mich, fast wie das Denken selbst." 59

Der Computer wird hier zum „Instrument"60 gleichzeitiger Improvisation und Notation, wobei es gerade die Möglichkeiten des Eingreifens in das Geschriebene sind, das Löschen und Kopieren, die diese Schreibweise zu ermöglichen scheinen und ironischerweise gerade dadurch wiederum "Allmachtsgefühle"61 einer "Autorin" evozieren: "Man fühlt sich dabei wie ein Gott, der etwas erschaffen kann und es wieder wegräumt, im selben Arbeitsgang"62 Gleichzeitig steht auch die Suggestion des göttlich Schöpferischen infrage, wenn sich das Verhältnis von Schreibender und Schreibgerät in einer anderen Interviewsequenz in einen Ritus verschiebt: "Das wird dann eine Art Tabernakel, es bekommt einen Fernsehcharakter und eine herausragende Wichtigkeit, wenn die Schrift auf dem Bildschirm erscheint. Für mich befördert der Computer auch das Spiel mit der Sprache, mit den Worten."63

58 Koelbl, Herlinde: Im Schreiben zu Haus, S. 64. 59 Niermann, Ingo: ELFRIEDE JELINEK. In: Fiktion: http://fiktion.cc/elfriede-jelinek/. (31.01.2021).

60 Becker, Peter von: Berg von Leichen und Schmerz, S. 6. 61 Ebd.

62 Niermann, Ingo: ELFRIEDE JELINEK.

63 Becker, Peter von: Berg von Leichen und Schmerz, S. 6.
Was diese selbstinszenatorischen Interviewäußerungen vorstellen, ist eine Verschiebung vom Notizenmachen hin zum Notieren im Fluxus ${ }^{64}$ der medialen Schreibpraxis: Die Notiz als materieller Ausdruck eines Gedankens auf scheinbar herumliegendes Schreibmaterial, wie eben ausdrücklich "alte" Briefumschläge, bricht in gewisser Weise aus der organisch-technischen Notation heraus, um dann möglicherweise wieder Teil von ihr zu werden - wie auch die szenische Notiz den Fluss des Textes unterbricht und ihn damit für Vergangenes und Zukünftiges öffnet.

In Jelineks Textverfahren, so ließe sich hinsichtlich des Verhältnisses von, szenischen Stellen' und anderem Text versuchsweise resümieren, treffen die verstimmlichten Positionen des scriptors, der nur abschreibt, des compilators, der von anderen etwas hinzufügt, des commentators, der von sich etwas hinzufügt, um den Text verständlich zu machen, und des auctors, der zwar eigene Gedanken ausdrückt, sich dabei auf andere Autoritäten stützt, ${ }^{65}$ aufeinander und kommen dabei gerade nicht zusammen. Vielmehr spielen sie sich auf und gegeneinander aus: Dynamiken von Macht, Ohnmacht und Zufall66 drängen gleichzeitig an die Oberfläche der Partitur eines heterophonen Stimmlichen, sodass am Ende vielleicht nur eine - einmal mehr unter der Mitarbeit von Barthes zu formulierende - Anweisung am Anfang dieser Versuchsanordnungen steht: horchen! nicht gehorchen.

\section{Literaturverzeichnis}

Annuß, Evelyn (2005): Theater des Nachlebens. München: Wilhelm Fink.

Barthes, Roland (2005): Vom Werk zum Text, übers. v. Dieter Hornig. In: ders.: Das Rauschen der Sprache. Frankfurt am Main: Suhrkamp 2005.

Barthes, Roland (2008): Die Vorbereitung des Romans, Frankfurt am Main: Suhrkamp.

Barthes, Roland (2010): Über mich selbst. Berlin: Matthes und Seitz.

$64 \mathrm{Vgl}$.: Elfriede Jelinek Forschungszentrum: Interview mit Sybille Krämer. https://www.youtube.com/watch?v=P5DUxMzKXyk. (31.01.2021).

65 Vgl.: Lindorfer, Bettina (1998): Roland Barthes. Zeichen und Psychoanalyse. München: Wilhelm Fink, S. 43.

$66 \mathrm{Vgl}$.: Elfriede Jelinek Forschungszentrum: Eine Zwischenbilanz: Kommentar von Clemens Risi zu „Notation und Aufführung". https://www.youtube.com/watch?v=vMGARV_Xw-4 \&feature=emb_logo. (31.01.2021). 
Becker, Peter von (1992): Wir leben auf einem Berg von Leichen und Schmerz. In: Theater heute - Gespräch mit Elfriede Jelinek, 1992/9.

De Mazza, Ethel Matala/Vogl, Joseph (2017): Graduiertenkolleg "Literatur- und Wissensgeschichte Kleiner Formen". In: Zeitschrift für Germanistik, 27/3, S. 579-585. JSTOR: www.jstor.org/stable/26583261. (31.01.2021).

Deleuze, Gilles (2000): Stotterte er ..., übers. v. Joseph Vogl. In: ders.: Kritik und Klinik. Frankfurt am Main, S. $145-154$.

Elfriede Jelinek-Forschungszentrum: Eine Zwischenbilanz: Kommentar von Clemens Risi zu "Notation und Aufführung". https://www.youtube.com/ watch?v=vMGARV_Xw-4\&feature $=$ emb_logo. (31.01.2021).

Elfriede-Jelinek-Forschungszentrum: Interview mit Sybille Krämer. https://www.youtube.com/ watch?v=P5DUxMzKXyk. (31.01.2021).

Ette, Ottmar (2016): Roland Barthes zur Einführung. Hamburg: Junius, ebook.

Folkerts, Hendrik (2014): documenta 14: https://www. documenta14.de/de/south/464_der_partitur_nach_ notation_verkoerperung_und_liveness (23.03.2021).

Heinrichs, Hans-Jürgen (2004): Gespräch mit Elfriede Jelinek. In: Sinn und Form 2004/6, S. 760-783.

Janke, Pia (2002): Die Nestbeschmutzerin. Jelinek und Österreich. Salzburg: Jung und Jung.

Janke, Pia (Hg.): Jelinek Handbuch. Stuttgart/Weimar: J.B. Metzler, S. 228-236.

Jelinek, Elfriede (1982): Burgtheater. Elfriede Jelineks Website: https://www.elfriedejelinek.com/fburgth1. htm. (31.01.2021).

Jelinek, Elfriede (2002a): Ein Sportstück. Reinbek bei Hamburg: Rowohlt.

Jelinek, Elfriede (2002b): Macht nichts. Eine kleine Trilogie des Todes. Reinbek bei Hamburg: Rowohlt.

Jelinek, Elfriede (2005): Ulrike Maria Stuart. Elfriede Jelineks Website: https://www.elfriedejelinek.com/ fstuart.htm. (31.01.2021).

Jelinek, Elfriede (2009): Abraumhalde. Elfriede Jelineks Website: https://www.elfriedejelinek.com/farhalde. htm. (31.01.2021).

Jelinek, Elfriede (2020): Schwarzwasser. Am Königsweg. Zwei Theaterstücke. Reinbek bei Hamburg: Rowohlt.

Jelinek, Elfriede: Das Kommen. ARD Audiothek: https:// www.ardaudiothek.de/hoerspiel-pool/01-lesungdas-kommen-von-elfriede-jelinek/78745986. (31.01.20121).

Jelinek, Elfriede: Das Licht im Kasten. Elfriede Jelineks Website: https://elfriedejelinek.com/flicht.htm. (31.01.2021).

Jelinek, Elfriede: Der Tod und Das Mädchen I-V. Berlin: BvT, S. 65.

Koelbl, Herlinde (1998): Im Schreiben zu Haus: wie Schriftsteller zu Werke gehen. Fotografien und Gespräche. München: Knesebeck.

Kormann, Eva (2009): Die Bühne als medialer Echo-Raum. Zu Elfriede Jelineks Bambiland. In: Schößler, Franziska (Hg.): Ökonomie im Theater der Gegenwart. Ästhetik, Produktion, Institution. Bielefeld: transcript.

Lehmann, Hans-Thies (1999): Postdramatisches Theater. Frankfurt am Main: Verlag der Autoren.

Lindorfer, Bettina (1998): Roland Barthes. Zeichen und Psychoanalyse. München: Wilhelm Fink.

Maresch, Rudolf (1993): Nichts ist verwirklicht. Alles muss jetzt neu definiert werden. In: ders. (Hg.): Zukunft oder Ende: Standpunkte, Analysen, Entwürfe. Berlin: Boer, S. 125-143.

Menke, Bettine (2001): Zitierfähigkeit: Zitieren als Exzitation. In: Gutenberg, Andrea/Poole, Ralph J. (Hg.): Zitier-Fähigkeit. Findungen und Erfindungen des Anderen. Berlin: Erich Schmidt, S. 153-171.

Nancy, Jean-Luc (1994): Die Kunst - Ein Fragment, übers. v. Jean-Pierre Dubost. In: ders. (Hg.): Bildstörung. Gedanken zu einer Ethik der Wahrnehmung. Leipzig: Reclam.

Nancy, Jean-Luc (2004): Zum Gehör. Zürich/Berlin: diaphanes.

Neubecker, Annemarie Jeanette (1992): Altgriechische Musik 1958-1986. In: Gärtner, Hans/Petersmann, Hubert (Hg.): Lustrum. Internationale Forschungsberichte aus dem Bereich des klassischen Altertums. Göttingen: Vandenhoeck \& Ruprecht.

Niermann, Ingo: ELFRIEDE JELINEK. In: Fiktion: http:// fiktion.cc/elfriede-jelinek/. (31.01.2021).

Palm, Kurt (1986): Elfriede Jelinek. In: ders. (Hg.): Burgtheater. Zwölfelläuten. Blut. Besuchszeit. Vier österreichische Stücke. Berlin: Hentschel, S. 227-233.

Samoyault, Tiphaine (2015): Roland Barthes: Die Biographie. Frankfurt am Main: Suhrkamp.

Štajner, Tamara (2012): Musiker sind Interpreten, Schriftsteller schaffen etwas. Elfriede Jelinek Forschungszentrum: http://www.elfriede-jelinekforschungszentrum.com/wissenschaftsportale/ musik/das-projekt/interview-mit-elfriede-jelinek/. (31.01.2021).

Stemann, Nicolas: Das ist mir sowas von egal! Wie kann man machen sollen, was man will? - Über die Paradoxie, Elfriede Jelineks Theatertexte zu inszenieren. In: Theater heute. Arbeitsbuch 2006, S. 62-68.

Weinbuch, Isabell (2010): Das musikalische Denken und Schaffen Carl Orffs. Ethnologische und Interkulturelle Perspektiven. Mainz u. a.: Schott Campus.

Wirth, Uwe (2010): Lob der Oberfläche! Der Tod und die Mode in Elfriede Jelineks, Jackie'. In: Eder, Thomas/ Vogel, Juliane (Hg.): Lob der Oberfläche. Zum Werk von Elfriede Jelinek. München: Wilhelm Fink.

Wörner, Karl H. (1980): Geschichte der Musik. Ein Studien- und Nachschlagewerk. Siebte Auflage. Göttingen: Vandenhoeck \& Ruprecht.

Wörner, Karl H. (1993): Geschichte der Musik. Ein Studien- und Nachschlagewerk. 8. Aufl., bearb. v. Wolfgang Gratzer u. a. Göttingen: Vandenhoeck \& Ruprecht. 